\title{
Adult age differences in subjective responses to dynamic socioemotional incentives
}

\author{
Sade J. Abiodun ${ }^{1}$, Galen A. McAllister ${ }^{1}$, Gregory R. Samanez-Larkin ${ }^{1,2,3}$, Kendra L. \\ Seaman ${ }^{4,5}$, \\ ${ }^{1}$ Center for Cognitive Neuroscience, Duke University \\ ${ }^{2}$ Center for the Study of Aging and Human Development, Duke University \\ ${ }^{3}$ Department of Psychology and Neuroscience, Duke University \\ ${ }^{4}$ Center for Vital Longevity, The University of Texas at Dallas \\ ${ }^{5}$ School of Behavioral and Brain Sciences, The University of Texas at Dallas
}

\begin{abstract}
Author note:
S.J.A is now at Princeton University. G.A.M is now at Tonic. This research and S.J.A. were supported by a pilot grant from P30-AG034424, K.L.S. was supported by T32AG000029, and G.R.S-L. was supported by R00-AG042596. Project details and analysis scripts available here: https://osf.io/3bzwt/ Thank you Galen A. McAllister for developing the initial versions of the stimuli used in this study. Correspondence should be addressed to: Sade J. Abiodun, fabiodun@princeton.edu
\end{abstract}




\begin{abstract}
Facial expressions are powerful communicative social signals that motivate feelings and action in the observer. However, research on incentive motivation has overwhelmingly focused on money and points and the limited research on social incentives has been mostly focused on responses in young adulthood. Previous research on the age-related positivity effect and adult age differences in social motivation suggest that older adults might experience higher levels of positive arousal to socioemotional stimuli than younger adults. Affect ratings following dynamic emotional expressions (anger, happiness, sadness) varying in magnitude of expression showed that higher magnitude expressions elicited higher arousal and valence ratings. Older adults did not differ significantly in levels of arousal when compared to younger adults, however their ratings of emotional valence were significantly higher as the magnitude of expressions increased. The findings provide novel evidence that socioemotional incentives may be relatively more reinforcing as adults age. More generally, these dynamic socioemotional stimuli that vary in magnitude are ideal for future studies of more naturalistic affect elicitation, studies of social incentive processing, and use in incentive-driven choice tasks.
\end{abstract}

Word Count: 3652

Keywords: naturalistic, faces, socioemotional stimuli, aging, emotion 


\section{Introduction}

Many of our interactions involve real-time social feedback. At all stages of life, the ability to perceive and react to social cues is integral for social reasoning and human development (Gluckman \& Johnson, 2013). Facial expressions are one of the earliest and most common forms of social communication, and our ability to process faces develops at an early age (Pascalis et al., 2012). Thus, faces are ideal experimental stimuli due to their empirical and ecological validity. In previous studies using face stimuli, researchers identified adult age differences in the ability to distinguish different emotional expressions (Ruffman et al., 2008). Studies have also highlighted the importance of using age-compatible stimuli in face-processing research and showed that certain variations of stimuli (such as age and gender) can significantly impact evaluation of expression and emotion (Ebner et al., 2010). More recent research using dynamic emotional expressions shows that use of more naturalistic stimuli enhances performance in older adulthood and minimizes age differences in emotion recognition (Holland et al., 2019). More generally, positive and negative emotional facial expressions can act as social rewards and punishments that may differentially motivate people across adulthood (Rademacher et al., 2014). Given how common facial expressions are as naturalistic incentives, the development of valence-specific social incentives could be useful for future studies of decision making.

Studies of decision making often examine choices between monetary gains and losses, while domains such as social incentives are less frequently used (Abeler \& Nosenzo, 2015; Xie et al., 2015). Social incentives provide a unique avenue to evaluate how individuals contextualize their choices based on their interactions with others (Izuma et al., 2008). In particular, social incentives may carry more relevance than monetary incentives across adulthood, as older adults often focus more on socioemotional goals than younger adults (Carstensen, 1992; Samanez-Larkin et al., 2009). Studies comparing hypothetical monetary and social incentives (i.e., time spent with social partners) have revealed greater adult age differences in social compared to monetary decision making (Seaman et al., 2016; Seaman et al., 2020) with older adults appearing to be more motivated by social than monetary rewards.

Numerous studies have examined differences in social information processing in older adults (Bailey et al., 2018; Roalf et al., 2011). These studies demonstrate age differences in the evaluation of positive vs. negative. This difference is most commonly attributed to socioemotional selectivity theory (SST) which suggests that differing perceptions of time horizons between older and younger adults' results in attentional and memory biases, specifically skewed (for older adults) towards positive, emotionally beneficial information (Carstensen, 1992). This well-documented age-related positivity effect has been hypothesized to be a method of "maintaining" positive affective states in older age and maximizing well-being in the moment (Kennedy et al., 2004; Reed, 2012; Samanez-Larkin et al., 2009). With regards to the perception of social stimuli, the agerelated positivity effect could contribute to significant variations in the perception and identification, and response to emotional cues. Examining age-related diversity in social 
incentive processing is important for more generalizable understanding of social perception across adulthood.

Monetary stimuli have been used as the incentive of choice by researchers for a number of reasons. They are easily manipulatable, numerically quantifiable, and have a clear demarcation between gain and loss domains. Social stimuli, on the other hand, are more complex, and subject to higher levels of context-dependent variability in perception and preference (Barrett et al., 2011). Properly comparing incentive domains would require development of social stimuli that operate similarly to monetary stimuli in a choice task: clear positive (gain) vs. negative (loss) domains, variation in reward magnitude, and tangible value. Such a stimuli set would allow for the interchangeable comparison of incentive processing in either social or monetary domains, opening the door to a deeper understanding of learning and decision making from a more diverse, naturalistic, and generalizable perspective.

In this study we created and partially validated a set of dynamic socioemotional incentives. Using a previously validated emotional facial expression database (Ebner et al., 2010; Holland et al., 2019), we created modified dynamic, incremented social stimuli. These stimuli were evaluated in two ways: 1) testing whether they elicited the desired affective response in participants (positive vs. negative, moderated by magnitude) and 2) examining potential age differences in stimulus processing. Based on the-age-related positivity effect, we hypothesized that older adults might report stronger valence and arousal ratings especially for positive stimuli compared to younger adults.

\section{Method}

Participants. Two hundred and seven participants (age: $\mathrm{M}=49.04, \mathrm{SD}=16.74$, range $=$ 21-78 years old) were recruited for an online study using Qualtrics Panels. Participants were screened with a brief demographics and health survey to ensure the sample was comprised of healthy adults with no history of psychiatric/neurological illness. Age and gender quotas were implemented to ensure an equal distribution of men and women in each age category. One participant was excluded for falling outside the age criterion. Data was collected from two samples. The survey was comprised of a demographics questionnaire and an affective response rating task (described below). Survey presentation was identical for each of the samples, which have been combined in the reported analysis. Total survey time was approximately 30 minutes and was approved by the Duke University Institutional Review Board.

Incremented Dynamic FACES. Using Dynamic FACES (Holland et al., 2019), we created videos of three emotional expressions (anger, happiness, sadness) that each varied in magnitude of expression (low, medium, high). Using Morph Age (Morph Age, 2018) high spatial and temporal resolution static frames were exported to create still images of the evolving expression. The Morph Age exports went from neutral to full expression. To create low magnitude emotional expressions, the first $\sim 50 \%$ of stills were used. To create 
the medium magnitude emotional expression, the first $\sim 75 \%$ of expressions were used. All stills were used to create the high magnitude full expression videos. The exact number of frames used to create low and medium magnitude were adjusted for each emotion and face model based on preliminary pilot data such that the three videos looked distinct (differences between low, medium, and high were perceivable) and the low magnitude expressions were identifiable for their category and looked slightly more emotional than neutral. These still images were combined into new videos using Adobe Animate (Adobe Animate CC, 2018). Video names coincided with a FACE model identifier number, facial expression description, and final expression magnitude (for example, 006_a_full.mp4 describes a video of face model 006, morphing from neutral to full angry expression).

Affect Rating Task. Subjects viewed a total of 54 stimulus videos ( 3 emotional expressions with 3 magnitudes per emotion for 6 face models; Figure 1). At the beginning of the task, participants were instructed to first rate their arousal intensity on a seven-point Likert scale. Participants were instructed that their rating should pertain to their own arousal, rather than a rating of the perceived level of arousal of the stimulus model or a rating of sexual arousal. Participants also rated their valence intensity on a seven-point Likert scale.

Following data collection, subjective ratings for both arousal and valence scales were reverse coded for data analysis. Ratings were collapsed across the 6 face models to calculate mean ratings per subject for each emotion type and magnitude. Based on the age range of our sample (21-78), participants were separated into three age groups: Younger adults (range $=21-39$ yo, mean $=29.74, \mathrm{n}=69$ ) Middle-aged (range=39-58yo, mean $=48.88, \mathrm{n}=69$ ) and Older adults (range=59-78yo, mean=68.51, $\mathrm{n}=69$ ). Mean ratings were calculated for each emotion type (Angry, Happy, Sad), magnitude of expression (Low, Medium, Full) and age group (Younger, Middle, Older), and used to run a threeway ANOVA between emotion, magnitude, and age group.

\section{Results}

Arousal. There was a main effect of Emotion on arousal ratings, F(2, 354) = $86.960, \mathrm{p}<.001, \eta_{\mathrm{p}}{ }^{2}=0.329$, such that Happy expressions elicited higher arousal than Angry expressions $\left(\mathrm{t}=8.995\right.$, $\left.\mathrm{p}_{\text {bonf }}<.001\right)$ or Sad expressions $\left(\mathrm{t}=10.113\right.$, $\left.\mathrm{p}_{\text {bonf }}<.001\right)$ and Angry expressions elicited higher arousal ratings than Sad expressions $(t=2.710$, $\left.\mathrm{p}_{\text {bonf }}=.022\right)$. There was a main effect of Magnitude on arousal ratings, $\mathrm{F}(2,354)=$ $17.869, \mathrm{p}<.001, \eta_{\mathrm{p}}{ }^{2}=0.092$, such that Full expressions elicited higher arousal ratings than Medium expressions $\left(\mathrm{t}=5.366, \mathrm{p}_{\text {bonf }}<.001\right)$ or Low expressions $\left(\mathrm{t}=4.751\right.$, $\mathrm{p}_{\text {bonf }}<$ $.001)$, but there was not a significant difference in arousal elicitation between Medium and Low expressions $\left(\mathrm{t}=2.084\right.$, $\mathrm{p}_{\text {bonf }}=.116$. $)$ These main effects were qualified by an interaction between Emotion and Magnitude, $\mathrm{F}(4,708)=19.145, \mathrm{p}<.001, \eta_{\mathrm{p}}{ }^{2}=0.098$, such that arousal ratings increased with magnitude more for Happy expressions $(\mathrm{F}=$ 
$31.730, \mathrm{p}<.001)$ than Angry expressions $(\mathrm{F}=1.876, \mathrm{p}=.155)$ or Sad expressions $(\mathrm{F}=$ 3.091, $\mathrm{p}=.047$ ). There were no significant main effects or interactions with age (all $\mathrm{p}>$ $.05)$, suggesting that age groups did not differ in their arousal ratings by emotion category and/or magnitude.

Valence. There was a main effect of Emotion on valence ratings, $\mathrm{F}(2,382)=$ $388.262, \mathrm{p}<.001, \eta_{\mathrm{p}}{ }^{2}=0.670$, such that Happy expressions elicited more positive valence ratings in comparison to Angry expressions $\left(\mathrm{t}=18.633\right.$, $\left.\mathrm{p}_{\text {bonf }}<.001\right)$ and $\mathrm{Sad}$ expressions $\left(\mathrm{t}=18.932\right.$, $\left.\mathrm{p}_{\text {bonf }}<.001\right)$. Both Angry and Sad expressions elicited negative valence ratings and did not differ significantly $\left(\mathrm{t}=-3.088\right.$, $\left.\mathrm{p}_{\text {bonf }}=.007\right)$. There was a main effect of Magnitude on valence ratings, $F(2,382)=7.326, p<.001, \eta_{p}{ }^{2}=0.037$, such that Full expressions elicited higher valence ratings (in either the positive or negative direction) than Low expressions $\left(\mathrm{t}=-3.156\right.$, $\left.\mathrm{p}_{\text {bonf }}=.0006\right)$, but there was not a significant difference in valence between Full and Medium expressions $(t=-.447$, p bonf $>0.999)$. There was an interaction effect between Emotion and Magnitude, $F(4,764)=88.025, p<$ $.001, \eta_{\mathrm{p}}{ }^{2}=0.315$, such that Full, Happy expressions were rated significantly more positively than medium Happy expressions $\left(\mathrm{t}=-5.596, \mathrm{p}_{\text {bonf }}<.001\right)$, but no valence differences when comparing Full vs. Medium Angry $(\mathrm{t}=3.405$, p bonf $=.025)$ and Full vs. Medium Sad $\left(\mathrm{t}=2.914\right.$, $\left.\mathrm{p}_{\text {bonf }}=.131\right)$ expressions.

There was a significant interaction between Emotion and Age, $F(4,382)=12.807$, $\mathrm{p}<.001, \eta_{\mathrm{p}}{ }^{2}=0.118$, such that Happy expressions elicited more positive valence ratings in older adults than younger adults $\left(\mathrm{t}=4.915\right.$, $\left.\mathrm{p}_{\mathrm{bonf}}=<.001\right)$ but young and middle-aged adults did not differ $\left(\mathrm{t}=3.359\right.$, $\left.\mathrm{p}_{\text {bonf }}=.030\right)$ and middle-aged and older adults did not differ $\left(\mathrm{t}=-1.452, \mathrm{p}_{\mathrm{bonf}}>.05\right)$. There was also a three-way interaction between Magnitude, Emotion and Age, $F(8,764)=2.892, p=.004, \eta_{p}{ }^{2}=0.029$. Follow-up analyses showed variation by age groups in increased valence with increasing magnitude of expression. Higher magnitude Happy (positive) expressions elicited more positive valence ratings, while higher magnitude Sad and Angry (negative) expressions elicited more negative ratings. This effect was significant but relatively weakest in young adults ( $F=11.391$ for Happy, $F=6.982, p<.001, p=.001$ for Angry, $F=3.277, p=.041$ for Sad), stronger in middle age adults $(\mathrm{F}=26.532, \mathrm{p}<.001$ for Happy, $\mathrm{F}=23.028, \mathrm{p}<.001$ for Angry, $\mathrm{F}=$ 17.956, $\mathrm{p}<.001$ for Sad $)$, and most pronounced in older adults $(\mathrm{F}=74.286, \mathrm{p}<.001$ for Happy, $F=43.375, \mathrm{p}<.001$ for Angry $\mathrm{F}=28.016, \mathrm{p}<.001$ for Sad). Although older adults showed larger effects of magnitude on valence ratings for all three emotion categories, the largest age difference was for Happy expressions where older adults showed the strongest effects of magnitude on valence ratings compared to the other age groups.

\section{Discussion}

In this study we investigated adult age differences in responses to dynamic social stimuli, using a modified version of the existing Dynamic FACES database (Holland et al., 2019). Our main goal was to validate this stimulus set, using participant ratings of subsequent arousal and valence following stimulus presentation. Increasing magnitude 
from low to full expression resulted in higher arousal and valence ratings. Happy faces were, on average, rated as most arousing and most positive, with significant increases in arousal ratings with expression magnitudes for each emotion. Age did not significantly impact arousal ratings, however older adults rated Happy expressions more positively (higher valence) and Sad/Angry expressions more negatively (lower valence) than younger adults.

Results reveal significant variation in affect ratings across emotion category, magnitude, and age groups. Valence ratings (Fig 2.) showed consistency between perceived positive (Happy expressions) and perceived negative (Angry/Sad expressions) stimuli, indicating effective categorization of emotions. Positive stimuli were rated as more arousing overall than negative stimuli (Fig 2.), and higher magnitude Happy expressions also garnered higher overall ratings. This result is corroborated by previous studies on emotional empathy, which have shown that vivid emotional expressions elicits emotional reactivity in observers (Bailey et al., 2018).

Interestingly, there were effects of stimulus magnitude on both valence and arousal for Happy expressions but only valence ratings were modulated by magnitude for Sad and Angry expressions. Arousal ratings for Sad and Angry expressions were moderately high across all magnitudes. It is possible that the thresholds created for low and medium magnitudes were set to high but the variation in valence ratings for these emotions suggests that they were well calibrated. Another possibility is the presence of a potential base threshold for reaction to negative expressions, which is less dependent on variations in the emotional intensity. This could be explained by the innate perception of anger as it pertains to threat detection. Previous research has shown that adults react to and register negative emotions (such as anger) more rapidly than other emotions, likely due to the evolutionary benefits of early threat recognition (Mather \& Knight, 2006).

There were no significant age differences in arousal ratings, contrary to our prediction that older adults would be more aroused by specific emotional expressions (Carstensen, 1992; Samanez-Larkin et al., 2009). However, the role of age as a moderator of valence ratings is consistent with our hypothesis that older adults would rate Happy expressions more positively than their younger counterparts. Older adults' ratings of happy faces increased in positivity between expression magnitudes more than younger adults (Fig 2.). This is consistent with the age-related positivity effect, which posits that older adults are more impacted by positive stimuli, and tend to dwell less on negative stimuli (Isaacowitz et al., 2006; Reed, 2012; Samanez-Larkin et al., 2009).

There are several limitations. First, the manipulation of any naturalistic stimuli set (especially one with dynamic components) requires accounting for the extent to which the audience is able to interpret and believe the presented information. Here, we generated dynamic images from a previously static set, demanding some mechanical and unnatural manipulation of expressions into their desired final magnitudes. This manipulation could affect participants reaction to the stimuli, especially at lower magnitudes (when the facial expressions were most contrived). To account for this, we included an additional question which asked half the participants how "natural" they 
found a given stimulus to be. While analysis of these naturalness scores revealed that lower magnitudes were rated as "less natural", there was not a significant main effect of expression magnitude on naturalness ratings, nor any notable age group differences, thus we do not believe that the arousal and valence ratings were significantly impacted by naturalness. An additional critical limitation is that all of the face models in this set are White, German adults. Although there is age and basic gender diversity, these stimuli lack racial and cultural diversity. Their utility may be limited with other racial groups for whom perception of emotional expressions in out-groups may differ compared to ingroups (Natu et al., 2011; Zebrowitz et al., 2010). This is a serious limitation of the present stimuli. However, the procedures used here could be applied to any stimulus set to create similar dynamic stimuli. We are currently unaware of any stimulus set that contains naturalistic high-resolution images of several emotions in adults of different ages from different racial/ethnic groups. The creation of such a resource would be of great benefit to the field. An alternative strategy would be to use synthetic stimuli to create maximum diversity among stimuli. Computer-generated imagery are becoming more and more lifelike and offer control to create even more dynamic and lifelike changes in expression (Chen et al., 2020).

Taken together, these findings demonstrate potential for using incremented dynamic socioemotional stimuli to elicit affective responses. Emotion categorization and incrementation of stimuli by level of expression (low, medium, full) was not only perceived by participants, but elicited the expected distinct reactions. This partially validates these stimuli for potential use in studies of choice preference and decision making. A future step would be comparing these same stimuli in a study with monetary rewards to evaluate potential incentive domain differences in social or monetary decision making. 
Figure 1.

Sample of face models and the magnitudes of expressions participants were shown (examples for Happy, Sad, and Angry expressions). After each video, participants provided arousal and valence ratings on a Likert scale (right). Videos were adapted from dFACES Database, which is available online at https://faces.mpdl.mpg.de/imeji/
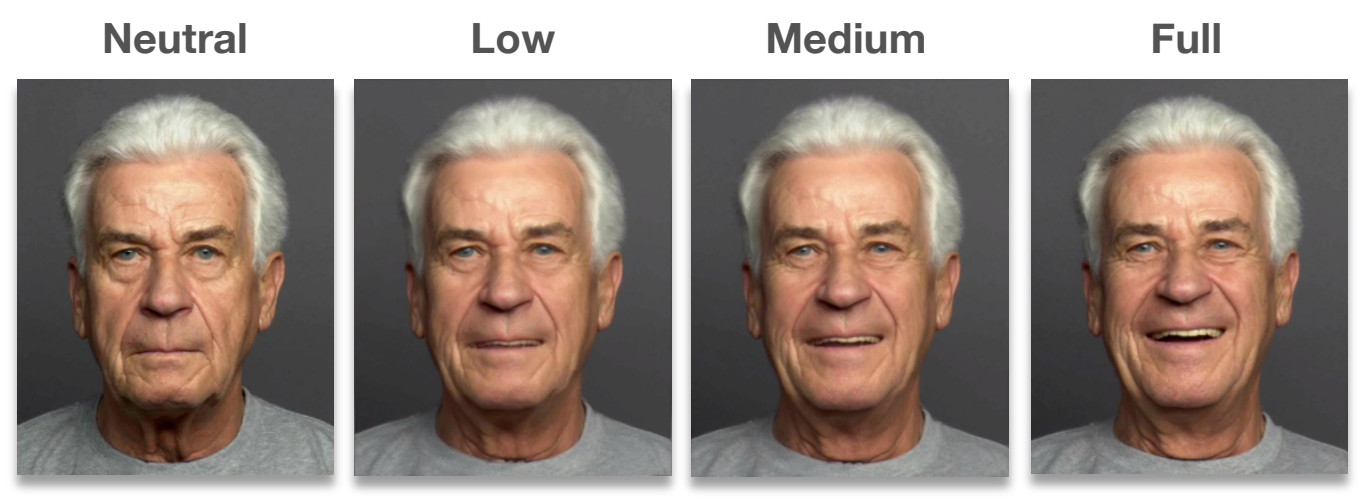

Arousal Rating
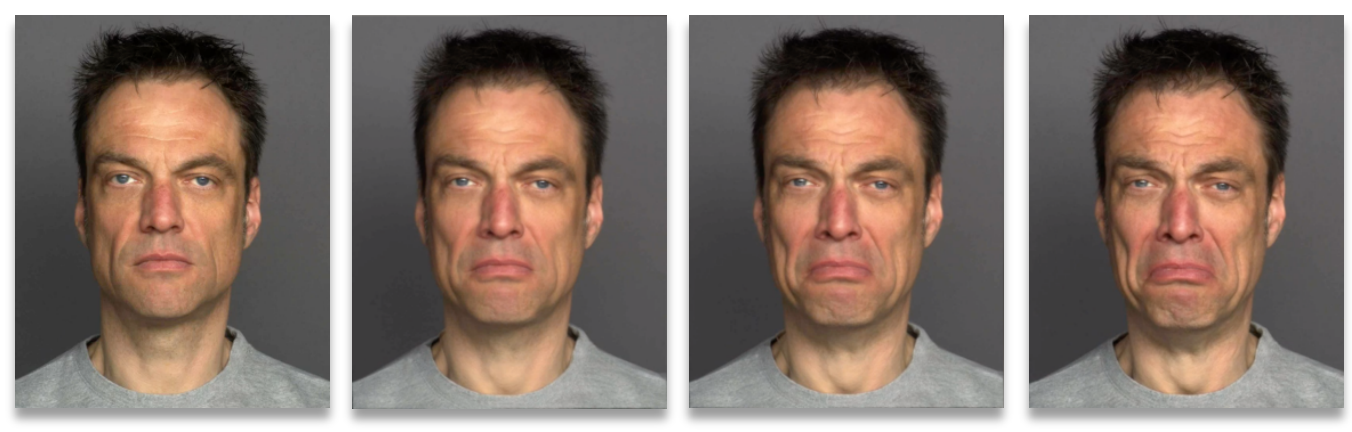

1 - Not At All Aroused

2

3

4

5

○ 6

7 -Very Aroused
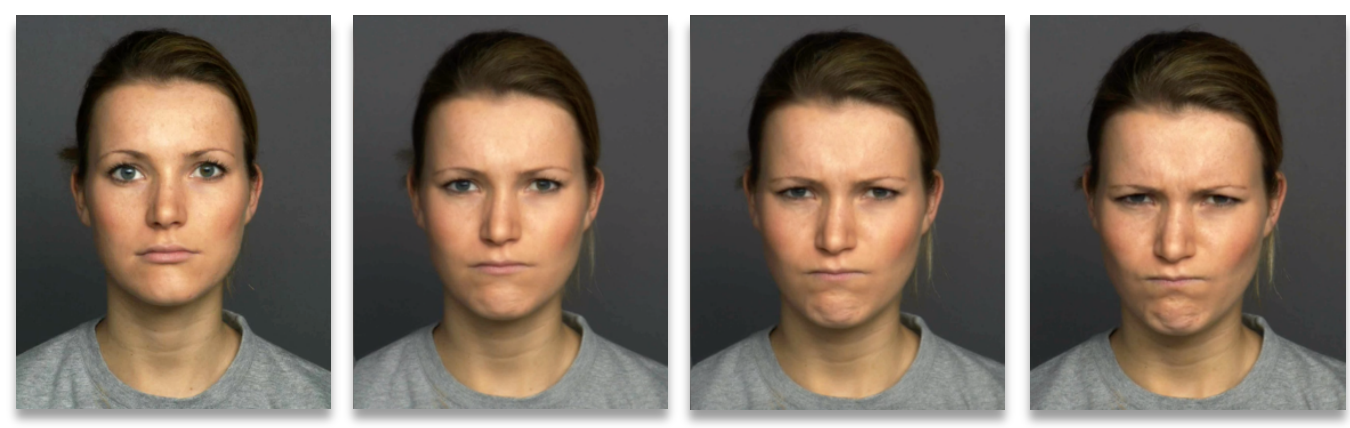

Valence Rating

1 - Very Negative

2 - Moderately Negative

3 - Slightly Negative

4 - Neutral

5 - Slightly Positive

6 - Moderately Positive

7 - Very Positive 
Figure 2.

Mean ratings for arousal $(1=$ not at all aroused, $7=$ very aroused $)$ and valence $(1=$ very negative, $4=$ neutral, 7 = very positive) by emotion category and expression magnitude for each age group. Happy expressions were most arousing overall, and arousal intensity increased with magnitude of the Happy expression but not for Sad or Angry expressions. Happy expressions also elicited positive valence ratings ( $>4)$, while Sad and Angry expressions elicited negative valence ratings $(<4)$. Ratings also increased in positive or negative valence across magnitudes of expression, but this effect was strongest in older adults especially for Happy expressions.

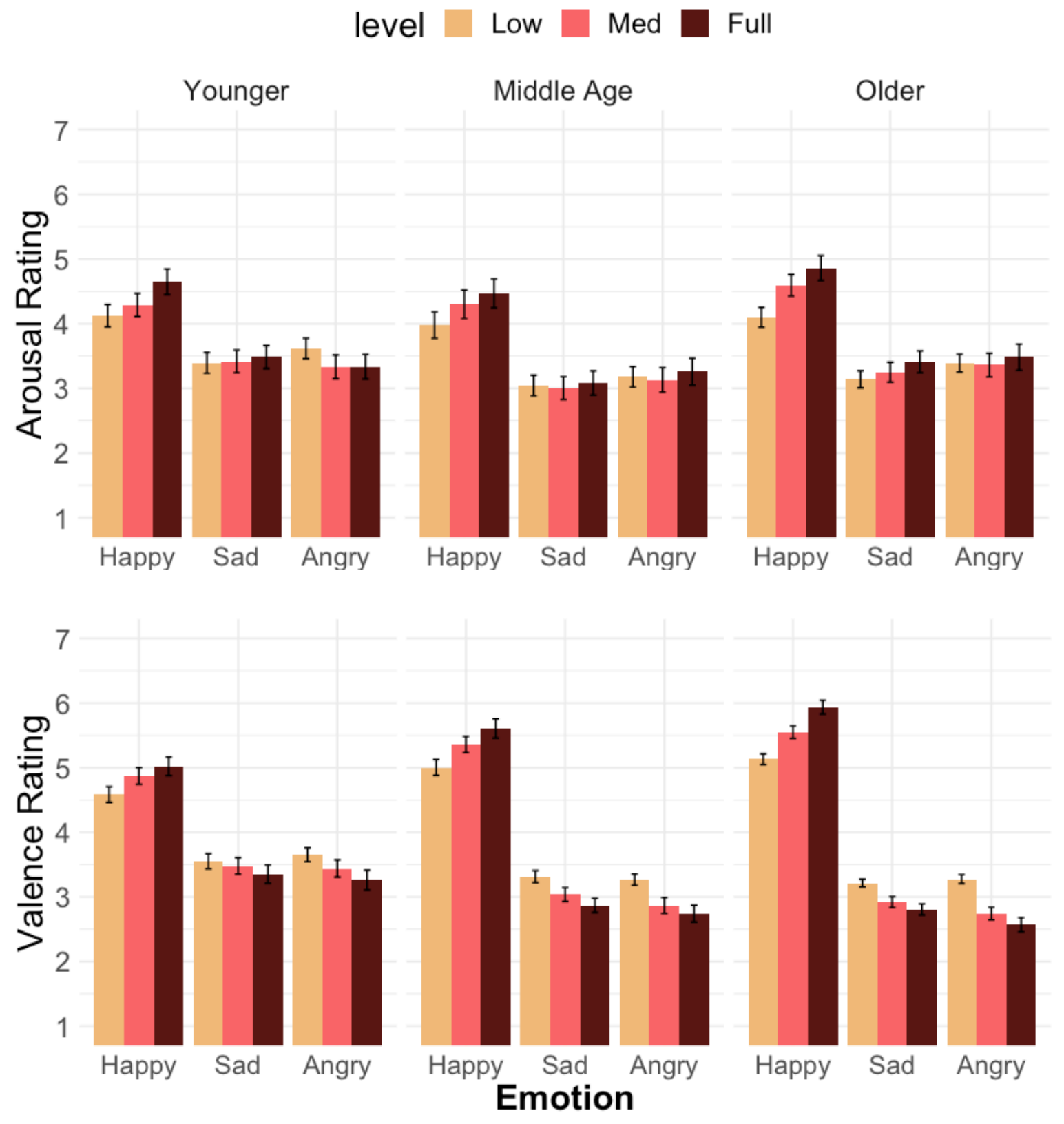




\section{References}

Abeler, J., \& Nosenzo, D. (2015). Self-selection into laboratory experiments: Pro-social motives versus monetary incentives. Experimental Economics, 18(2), 195-214. https://doi.org/10.1007/s10683-014-9397-9

Adobe Animate CC (Version 18). (2018). [Computer software]. Adobe Systems. http://adobe.com/products/animate.html

Bailey, P. E., Brady, B., Ebner, N. C., \& Ruffman, T. (2018). Effects of Age on Emotion Regulation, Emotional Empathy, and Prosocial Behavior. The Journals of Gerontology: Series B. https://doi.org/10.1093/geronb/gby084

Barrett, L. F., Mesquita, B., \& Gendron, M. (2011). Context in Emotion Perception. Current Directions in Psychological Science, 20(5), 286-290. https://doi.org/10.1177/0963721411422522

Carstensen, L. L. (1992). Social and Emotional Patterns in Adulthood: Support for Socioemotional Selectivity Theory. 8.

Chen, C., Messinger, D., Duan, Y., Ince, R. A., Garrod, O. G. B., schyns, P., \& Jack, R. (2020). Dynamic facial expressions of emotion decouple emotion category and intensity information over time [Preprint]. PsyArXiv. https://doi.org/10.31234/osf.io/4gpev

Ebner, N. C., Riediger, M., \& Lindenberger, U. (2010). FACES-A database of facial expressions in young, middle-aged, and older women and men: Development and validation. Behavior Research Methods, 42(1), 351-362. https://doi.org/10.3758/BRM.42.1.351

Gluckman, M., \& Johnson, S. P. (2013). Attentional capture by social stimuli in young infants. Frontiers in Psychology, 4. https://doi.org/10.3389/fpsyg.2013.00527 
Holland, C. A. C., Ebner, N. C., Lin, T., \& Samanez-Larkin, G. R. (2019). Emotion identification across adulthood using the Dynamic FACES database of emotional expressions in younger, middle aged, and older adults. Cognition and Emotion, 33(2), 245-257. https://doi.org/10.1080/02699931.2018.1445981

Isaacowitz, D. M., Wadlinger, H. A., Goren, D., \& Wilson, H. R. (2006). Selective preference in visual fixation away from negative images in old age? An eyetracking study. Psychology and Aging, 21(1), 40-48.

https://doi.org/10.1037/0882-7974.21.1.40

Izuma, K., Saito, D. N., \& Sadato, N. (2008). Processing of Social and Monetary Rewards in the Human Striatum. 11.

Kennedy, Q., Mather, M., \& Carstensen, L. L. (2004). The Role of Motivation in the AgeRelated Positivity Effect in Autobiographical Memory. 15(3), 7.

Mather, M., \& Knight, M. R. (2006). Angry Faces Get Noticed Quickly: Threat Detection is not Impaired Among Older Adults. The Journals of Gerontology Series B: Psychological Sciences and Social Sciences, 61(1), P54-P57. https://doi.org/10.1093/geronb/61.1.P54

Morph Age (4.2.5). (2018). [Computer software]. Creaceed. https://creaceed.com/morphage/about

Natu, V., Raboy, D., \& O’Toole, A. J. (2011). Neural correlates of own- and other-race face perception: Spatial and temporal response differences. NeuroImage, 54(3), 2547-2555. https://doi.org/10.1016/j.neuroimage.2010.10.006

Pascalis, O., Tanaka, J. W., \& Lee, K. (2012). Development of Face Processing. 15. 
Rademacher, L., Salama, A., Gründer, G., \& Spreckelmeyer, K. N. (2014). Differential patterns of nucleus accumbens activation during anticipation of monetary and social reward in young and older adults. Social Cognitive and Affective Neuroscience, 9(6), 825-831. https://doi.org/10.1093/scan/nst047

Reed, A. E. (2012). The theory behind the age-related positivity effect. Frontiers in Psychology, 9.

Roalf, D. R., Mitchell, S. H., Harbaugh, W. T., \& Janowsky, J. S. (2011). Risk, Reward, and Economic Decision Making in Aging. 10.

Ruffman, T., Henry, J. D., Livingstone, V., \& Phillips, L. H. (2008). A meta-analytic review of emotion recognition and aging: Implications for neuropsychological models of aging. Neuroscience \& Biobehavioral Reviews, 32(4), 863-881. https://doi.org/10.1016/j.neubiorev.2008.01.001

Samanez-Larkin, G. R., Robertson, E. R., Mikels, J. A., Carstensen, L. L., \& Gotlib, I. H. (2009). Selective Attention to Emotion in the Aging Brain. Psychology and Aging, 24(3), 519-529. https://doi.org/10.1037/a0016952

Seaman, Kendra L., Gorlick, M. A., Vekaria, K. M., Hsu, M., Zald, D. H., \& SamanezLarkin, G. R. (2016). Adult age differences in decision making across domains: Increased discounting of social and health-related rewards. Psychology and Aging, 31(7), 737-746. https://doi.org/10.1037/pag0000131

Seaman, Kendra Leigh, Juarez, E., Troutman, A., Salerno, J., Samanez-Larkin, S., \& Samanez-Larkin, G. R. (2020). Decision making and mental health across adulthood during social distancing [Preprint]. PsyArXiv. https://doi.org/10.31234/osf.io/dr798 
Xie, W., Yan, C., Ying, X., Zhu, S., Shi, H., Wang, Y., Cheung, E. F. C., \& Chan, R. C. K. (2015). Domain-specific hedonic deficits towards social affective but not monetary incentives in social anhedonia. Scientific Reports, 4(1), 4056. https://doi.org/10.1038/srep04056

Zebrowitz, L. A., Kikuchi, M., \& Fellous, J.-M. (2010). Facial resemblance to emotions: Group differences, impression effects, and race stereotypes. Journal of Personality and Social Psychology, 98(2), 175-189.

https://doi.org/10.1037/a0017990 